\title{
Experiencias de Inclusión-Exclusión de un Grupo de Sordos Usuarios de la Lengua de Señas Mexicana
}

\author{
Inclusion-Exclusion Experiences of a Group of Deaf People \\ Users of the Mexican Sign Language
}

\author{
Judith Pérez-Castro * \\ Johan Cristian Cruz-Cruz
}

Universidad Nacional Autónoma de México, México

\begin{abstract}
En este artículo, analizamos las experiencias de inclusión y exclusión social de un grupo de personas Sordas, usuarias de la Lengua de Señas Mexicana (LSM). Partimos del debate sobre el reconocimiento de los Sordos como comunidad para, posteriormente, discutir los principales modelos implementados en su atención educativa. La perspectiva metodológica fue de tipo cualitativa; específicamente, utilizamos el método de la historia oral temática. El trabajo empírico consistió en la realización de entrevistas a un grupo Sordos que, en su mayoría, estaba estudiando la educación superior. Los resultados nos mostraron que aquellos que crecieron en una familia de Sordos, no se asumen como personas con discapacidad, lo que a su vez marca diferencias en la forma en que se relacionan con la LSM y con el español. Estas personas tienden a enfrentar múltiples barreras a lo largo del sistema educativo como la débil formación académica, la carencia de intérpretes de LSM, la poca capacitación de los profesores y la falta de información sobre la oferta académica accesible. Concluimos que es fundamental implementar políticas a largo plazo, que recuperen el conocimiento experto de las personas Sordas y disminuyan los factores que incrementan el riesgo de exclusión.
\end{abstract}

Descriptores: Sordos; Exclusión social; Barreras; Facilitadores.

In this paper, we analyze the inclusion and exclusion experiences of a group Deaf people, users of Mexican Sign Language (LSM). We begin with the discussion about the acknowledgement of Deaf people as a community and, after that, we present some of the most important educational models directed to this group of the population. It was a qualitative research, based on the thematic oral history. For the empirical work, we conducted interviews with a group of Deaf people who were studying higher education. The results showed us that those who grew up in a family of Deaf people do not see themselves as persons with disabilities and this, in turn, makes differences in their relationship with the LSM and with the Spanish language. These individuals tend to face different barriers throughout the educational system, such as the feeble academic background, a lack of LSM interpreters, poor teacher training, and a lack of information about accessible academic offering. We conclude that it is important to implement long-term policies, based on the expert knowledge of Deaf people, to counter the factors that increase the risk of exclusion of this population.

Keywords: Deaf people; Social exclusion; Barriers; Enablers. 


\section{Introducción}

La exclusión es un fenómeno que remite a la disolución de los vínculos con la sociedad, reduce las oportunidades individuales o colectivas, impide la participación plena y limita el acceso a los sistemas de bienestar y protección social (Blanco, 2008). De acuerdo con Castel (2004), históricamente ha habido tres formas de exclusión: la primera conlleva la sustracción completa de un grupo social a través de la deportación, el destierro o el exterminio; la segunda refiere a la separación de un sector de la población por medio del encierro en cárceles, manicomios, guetos o leprosarios; y la tercera consiste en la atribución de una condición excepcional a determinados grupos que, aunque les permite permanecer en la comunidad, acota el ejercicio de sus derechos o restringe su participación, como ha ocurrido con los pueblos indígenas, los judíos y los afrodescendientes.

Si bien el autor reconoce que, en las sociedades actuales, es poco probable que podamos encontrar casos concretos de las dos primeras, la tercera forma de exclusión aún prevalece, como resultado de "la profunda ambigüedad de las políticas de discriminación positiva en las que podemos incluir la mayoría de las políticas de inserción [...] este tratamiento social estigmatiza muchas veces a las poblaciones implicadas" (Castel, 2004, p. 69). La situación de la Comunidad de Sordos es un ejemplo de este tipo de exclusión porque, al igual que la población con discapacidad, han sido vulnerados en sus derechos, sus oportunidades de desarrollo, su participación social y su bienestar individual, por considerarlos "deficientes", "incapacitados" o "anormales". Asimismo, a partir de la idea de normalidad y apoyándose el saber experto de campos como la medicina, la audiología, la foniatría, la psicolingüística y las ciencias cognitivas, entre otras, los Sordos han sido objeto de una serie de tipologías que los clasifican entre sordos profundos o hipoacúsicos, prelingüísticos o postlingüísticos, los que pueden recibir un implante coclear y los que no pueden, o bien, sordos que hablan y los que no hablan (Acosta, 2006; Cuevas 2013).

Pero, la exclusión es sólo una parte del fenómeno, es decir, no es una circunstancia absoluta, sino que los individuos solemos movernos a lo largo del continuum inclusiónexclusión (Subirats et al., 2005); incluso aquellos que tradicionalmente son considerados o se autodenominan excluidos están constantemente movilizando recursos, implementando estrategias, construyendo redes de relación y abriendo espacios para su participación (Karsz, 2004). Los Sordos no son la excepción y, desde de los años 70 del siglo pasado, han luchado por el reconocimiento de las lenguas de señas, así como por su estatus de comunidad(es).

Nuestro objetivo en este artículo es analizar las experiencias de inclusión y exclusión social de un grupo de personas Sordas, usuarias de la Lengua de Señas Mexicana (LSM). En los primeros apartados, presentamos el debate en torno a la Comunidad de Sordos, para después discutir algunas de las propuestas educativas desarrolladas para la atención de este sector de la población. Posteriormente, explicamos la ruta metodológica que nos permitió acercarnos a los sujetos de estudio y los principales resultados obtenidos a través de las entrevistas. Por último, hacemos algunas consideraciones generales sobre lo aquí expuesto. 


\section{Comunidad de sordos y lengua de señas}

Tradicionalmente, la sordera ha sido entendida como un problema físico, situando la atención en la (in)capacidad para percibir los sonidos. No obstante, desde la segunda mitad del siglo XX, esta postura ha sido fuertemente cuestionada, con el argumento de que al igual que ocurre con "el género, la edad, la raza y otras categorías similares, la diferencia física está involucrada, pero, dichas diferencias no tienen significados inherentes. Necesitan ser interpretadas y no pueden ser aprehendidas fuera de la red de significados creados culturalmente" (Baynton, 2006, p. 33).

Anteriormente, la sordera era atribuida a la herencia o el envejecimiento. En las antiguas culturas occidentales, como la Grecia clásica y el Imperio Romano, su ocurrencia era considerada una maldición porque impedía la comunicación con los otros. Los sordos que no habían aprendido a hablar eran ridiculizados, excluidos de la vida social, calificados como no inteligentes y personas sin estatus, incluso, en ocasiones, inferiores a los animales (Rose, 2006). "El lenguaje era el sello distintivo del logro humano, por lo que la mudez era más que una condición física. La incapacidad para hablar iba de la mano con la incapacidad de razonar, de la mano con la estupidez" (Rose, 2006, p. 21). De ahí, la constante de oralizar a los sordos, como una manera de romper su aislamiento.

De acuerdo con Davis (1995), hasta principios del siglo XVIII, los sordos estuvieron prácticamente invisibilizados de la vida social y, salvo algunas excepciones, tampoco hubo referencias sobre ellos en la literatura especializada. No obstante, durante la Ilustración, el tema de la sordera se fue abriendo espacio en la discusión académica. La dimensión filosófica introducida al debate en torno al significado de "ser humano", trajo consigo la idea de que, en el caso de los sordos, la dicotomía civilización versus barbarie podía superarse si éstos aprendían el lenguaje de la mayoría, lo que dio pie a las primeras escuelas formales en el siglo XVIII (Moores, 2010). Su educación se apoyó en el uso de las señas, el habla y la escritura, aunque, inicialmente, se le dio mayor énfasis a esta última. Pero, lo anterior no acalló la cuestión de la oralidad porque, desde algunas perspectivas, el habla era lo característico de la humanidad y privilegiar la escritura significaba atentar contra el orden natural (Moores, 2010).

La disputa entre las señas y el habla llegó a su punto más álgido durante el siglo XIX, resolviéndose finalmente a favor de la alfabetización oral de los sordos. En ello, la educación tuvo un papel fundamental, ya que se hicieron campañas continuas para eliminar la lengua de señas de los procesos de enseñanza (Baynton, 2006). En el siglo XX, el oralismo se consolidó en casi todos los sistemas educativos de Europa y América, a lo que también contribuyó el desarrollo de dispositivos tecnológicos para la audición.

Esta tendencia se mantuvo indemne hasta que, en la década los 70, los trabajos de William Stokoe y sus colaboradores cuestionaron por primera vez el paradigma oralista. Básicamente, lo que sostenían era que la lengua de señas y la hablada compartían diversas características, gramaticales, morfológicas y sintácticas y que, al igual que las palabras se construían a partir de fonemas que por sí mismos carecían de significado, las señas estaban formadas por partes que tampoco tenían sentido cuando se observaban de manera aislada (Barreto y Cortés 2014; Herrera et al., 2016). Todo esto fortaleció el movimiento de los Sordos que, apoyándose en el debate sobre los derechos, demandó el reconocimiento oficial de sus lenguas. 
El tema de los derechos no sólo fue una reivindicación de este colectivo social, sino que constituyó una exigencia central de los movimientos a favor de las personas con discapacidad. No obstante, los Sordos se separaron tempranamente de ellos, con el argumento de que el modelo social de discapacidad ponía demasiada atención en la accesibilidad, lo que, si bien favorecía a la inclusión, desde su perspectiva era insuficiente. Su meta era lograr el "verdadero respeto por la existencia de una realidad cultural Sorda [que condujera] al reconocimiento de aquellos asuntos de la Comunidad Sorda que quedan fuera del modelo social” (Ladd, 2011, p. 173).

Actualmente, la discusión sobre el reconocimiento de los Sordos como comunidad lingüística o como personas con discapacidad continúa vigente, tanto en su interior como entre los especialistas en el tema. Pero, además, los Sordos no conforman una sola comunidad propiamente dicha, sino varias de ellas, lo cual complejiza todavía más su situación. De este modo, hablar de "comunidad" a partir de un grupo heterogéneo, como los Sordos, apela a los elementos culturales que le dan sentido (Acosta, 2006); la lengua de señas tal vez sea el más importante, pero, a la par están otros, como la influencia mutua entre sus miembros, las normas y valores que comparten, las redes de apoyo y el reconocimiento de la diversidad que los distingue. Se trata, pues, de un colectivo socialmente complejo que cuestiona los modelos dominantes de ser y hacer en el mundo, y que necesitamos conocer más de cerca para entenderla desde su unicidad y diversidad.

\section{Modelos educativos para la atención de los sordos}

Los cambios de finales del siglo XIX y la primera mitad del XX tuvieron un papel determinante en las ideas que actualmente prevalecen en la educación para los Sordos. De acuerdo con Ladd (2011), aunque a lo largo del XIX el número de escuelas que utilizaban el método mix to había crecido significativamente, en las últimas dos décadas, se registró un alarmante retroceso debido a la importancia otorgada a la oralidad. Esta tendencia se nutrió de tres grandes fuentes, en primer lugar, el discurso cristiano que veía en la voz el espíritu que Dios le había otorgado al hombre. En segundo, los avances científicos, particularmente en el campo de la medicina. Hacia finales de la década de 1870, Galton apoyándose en las teorías darwinistas, empezó a sentar las bases para el estudio de la herencia, que lo condujeron al establecimiento de un estándar para la especie humana y, posteriormente, al desarrollo de lo que hoy se conoce como eugenesia (del Castillo, 2005; Davis, 2006). También, elabora su teoría antropométrica con la que intentó mejorar la raza humana y en la que proponía prohibir la reproducción de personas con "anormalidades", recurriendo a todos los métodos posibles, incluso la castración (Davis, 2006).

Una tercera fuente fue la celebración del Segundo Congreso Internacional de Maestros Sordomudos de 1880, también conocido como el Congreso de Milán. Aquí, se consolidó la idea de la normalización de los sordos a través de la oralización y se prohibió el uso de la lengua de señas (Herrera et al., 2016). La educación especial se consideró el agente más importante para este proceso, como sucedió con las personas con discapacidad y, al igual que ellas, los Sordos fueron definidos por sus rasgos negativos (Skliar, 1998), es decir, por aquello que los separaba de los oyentes.

Esta perspectiva predominó hasta la primera mitad del siglo XX, cuando el paradigma de la oralidad empezó a ser cuestionado. Los señalamientos vinieron de dos frentes, uno de 
ellos fue la lingüística, primordialmente, con las investigaciones de Stokoe que ya señalábamos. El otro fue la pedagogía, desde donde se objetaban los magros progresos de los sordos, quienes en su mayoría tenían un enorme rezago educativo o quedaban fuera del sistema de enseñanza (Saizarbitoria, 1999).

En este contexto, entre principios de los 70 y finales de los 90, surgieron varias propuestas; las más importantes fueron los denominados enfoques mixtos como la comunicación total, el bimodalismo y la educación bilingüe-bicultural. El primero propone el uso de todos los elementos de comunicación, ya sea lengua de señas, gestos, deletreo manual, materiales impresos o rehabilitación auditiva, con base en las necesidades de los estudiantes (Moores, 2010). Los defensores de la comunicación total la entienden como una filosofía, ya que no se apoya en un solo método o estrategia, sino en la incorporación de varios de ellos. Sin embargo, sus críticos han señalado que este método centró su atención fundamentalmente en la traducción del discurso oral al lenguaje de señas, lo cual "derivó en un amplio uso de lenguaje oral signado que difícilmente respetó la estructura propia del lenguaje de señas" (Becerra, 2008, p.108).

Por su parte, el modelo bimodal recurre a la combinación de expresiones orales con lengua de señas para la transmisión de las estructuras semánticas y sintácticas del lenguaje oral (Becerra, 2008). Esta propuesta se apoya en la traducción simultánea entre las dos lenguas; de ser necesario recurre a la modificación o creación de nuevos signos para la mejor representación de la lengua oral. Por esta razón, su implementación resulta bastante compleja, especialmente para los propios sordos, que se enfrentan al problema de dominar dos códigos lingüísticos, con estructuras y referentes propios (Becerra, 2008). Tanto el bimodalismo como la filosofía de la comunicación total han sido muy cuestionados debido al reforzamiento que han hecho del oralismo, a través de la combinación de las señas con la voz, o bien, de la mímica con materiales visuales.

El tercer modelo, la educación bilingüe-bicultural, le da a la lengua de señas el mismo estatus que a las demás, por lo que fomenta su uso como primera lengua, para después, introducir al Sordo a la lengua dominante de su cultura. Su punto de partida es el derecho de las personas Sordas a ser educados en su lengua natural (Becerra 2008); asimismo, dado que reconoce que éstas no poseen muchas habilidades en el lenguaje oral pone especial atención en los procesos de adquisición de la lengua y de la lectura. El trabajo con textos escritos y reales es una de sus estrategias de base porque constituyen un buen insumo de estructuras lingüísticas y vocabulario (Lissi et al., 2012).

Estos enfoques coexisten hasta el día de hoy, tanto en la educación especial como en la regular, con sus respectivas variantes y adaptaciones. Más recientemente, en el siglo XXI, ha ido cobrando fuerza la perspectiva de la co-educación (coenrollment) entre personas con baja audición, Sordos y oyentes, que consiste en el desarrollo de un programa bilingüe incorporado a la escuela convencional (Antia et al., 2019). La co-educación se diferencia de la integración porque, más allá de la copresencia física, busca que todos se sientan partícipes de las distintas actividades áulicas e institucionales, ya sean pedagógicas, interpersonales o sociales. En congruencia con lo que establece la Convención de los Derechos de las Personas con Discapacidad, propone la utilización de los medios, modos y lenguajes más adecuados, para favorecer al máximo el desarrollo académico y social de todos los estudiantes, en especial de los ciegos, sordos o sordociegos (Antia et al., 2019). Al momento, aún no logran verse los alcances de este enfoque que sin duda plantea múltiples retos, especialmente para las instituciones que atienden a la población más 
vulnerable o que no disponen de suficientes recursos para ofrecer condiciones educativas adecuadas.

\section{Método}

En este artículo, analizamos las experiencias de inclusión y exclusión social de un grupo de Sordos, usuarios de la LSM. El enfoque fue de tipo cualitativo y específicamente nos apoyamos en la historia oral temática, que es un método que busca reflexionar sobre el papel de la memoria colectiva, para reivindicar a los grupos invisibilizados por la historia oficial (Thompson, 1998). Esto permite dar voz a los que han sido excluidos de la dinámica social o han ocupado un papel periférico, enfrentando múltiples dificultades para el ejercicio de sus derechos, tener condiciones de vida digna y acceder a los servicios públicos básicos (Padilla, 2010; Martínez, 2012), como en el caso de la Comunidad de Sordos.

Para recoger los testimonios de los sujetos, elaboramos un guion abierto en el que consideramos los siguientes ejes: 1) Lengua de señas y comunicación; 2) Identidad sorda; 3) Trayectoria educativa; 4) Barreras y facilitadores; y 5) Motivaciones y expectativas.

\section{Características de la muestra}

El muestreo fue no probabilístico, de tipo intencional, para lo cual establecimos tres criterios: que fueran personas Sordas adultas, que usaran la LSM y que cuando menos tuvieran estudios de nivel medio superior. A través de la técnica de avalancha (MartínCrespo y Salamanca, 2007) se fueron ubicando y contactando a cada uno de los sujetos de la investigación.

Entrevistamos a seis $\mathrm{S} /$ sordos, cinco hombres y una mujer. Dos de ellos tenían entre 20 y 24 años, otros dos estaban entre 30 y 34, y los dos restantes tenían más de 35 años. Todos habían sido oralizados, pero, sólo cinco eran bilingües en LSM y en español; de ellos, dos tenían una competencia alta en la lengua de señas y tres tenían una competencia baja. El último, aunque la había aprendido, prácticamente no la utilizaba. Cuatro de los entrevistados nacieron en familias de Sordos y todos hicieron parte de su trayectoria escolar previa en la educación especial, en donde adquirieron o perfeccionaron la LSM. Finalmente, cuatro de ellos estaban en la educación superior, uno la había terminado y otro había estudiado hasta la preparatoria.

\section{Análisis de la información}

Para el análisis, se transcribieron las entrevistas y, a partir de una primera lectura, se ordenó la información con base en los ejes anteriormente señalados. En una segunda lectura, por cada una de las entrevistas, se identificaron categorías para los cinco ejes y se etiquetaron los fragmentos correspondientes. Por último, se hizo un análisis transversal de las entrevistas con el fin de encontrar temáticas en común o diferencias importantes entre ellas.

\section{Consideraciones éticas}

Para la dimensión ética de la investigación, nos apoyamos en la propuesta de Liamputtong (2010), que plantea que el trabajo con los sujetos de estudio debe basarse en el diálogo, la colaboración y principalmente el respeto a su integridad personal y cultural. De esta manera, para solicitar el consentimiento informado, a todos los participantes se les explicó el sentido y alcances de la investigación. También, se les aclaró que podían abstenerse de 
contestar las preguntas que les resultaran incómodas o detener la entrevista si lo creían pertinente. Asimismo, aunque la mayoría de los entrevistados era bilingüe, tenían diversos grados de comprensión del español, por ello, se les proporcionó el guion previamente para que lo revisaran y, cuando fue necesario, nos apoyamos en el intérprete de LSM a fin de que todas las preguntas quedaran totalmente claras.

\title{
5. Resultados
}

\subsection{La relación inclusion-exclusión desde la perspectiva de los sordos}

De acuerdo con Skliar (2012), la sordera es un territorio de representaciones que superan los límites de los modelos que socialmente hemos construido para explicarla. Estas ideas están mediadas por las relaciones que los Sordos establecen con otros Sordos y con los oyentes, y se concretan en formas diversas de comprender su condición, de interpretar su realidad y de actuar en los distintos ámbitos de la vida cotidiana. En nuestros entrevistados, son notables las diferencias entre aquellos que nacieron en una familia de Sordos y los que lo hicieron en una de oyentes.

\begin{abstract}
[... ] cuando yo nací no veía este tema de la sordera, o sea, para mí no existía esto de la sordera, yo me sentía normal, como cualquier otra persona en la calle, yo no tenía ningún conflicto en este aspecto. Pero, cuando tenía como cinco años, fue cuando me empecé a dar cuenta y me empezaron a platicar de mis bisabuelos, de mis abuelos, como de toda esta herencia. $\Upsilon$ pues sí, sentía como algo muy natural, muy normal, pero sí, fue cuando me di cuenta de que nosotros éramos sordos y que éramos distintos a los oyentes. (Entrevistado 1)
\end{abstract}

En contraste, está el testimonio de una persona que creció en una familia de oyentes:
To me siento una persona discapacitada, ya que no oigo y me es difícil comunicarme con las personas que pueden escuchar. He recibido terapia de lenguaje para poder hablar, pero, se me ha complicado mucho, no he podido aprender la habilidad de hablar. Mi familia es hablante y ellos escuchan muy bien y tratan de hacerme gestos y hablan un poco con la boca abierta para entender. Pero, algunos no conocen el poder hablar, no gesticulan bien, entonces, se me dificulta entenderlos. (Entrevistado 5)

Para los cuatro que nacieron en familias de Sordos, la sordera se vive como algo natural y no la ven como una limitación o deficiencia. En contraste, los dos que crecieron en familias de oyentes, se asumen como personas con discapacidad, con muchas dificultades en su vida cotidiana y con pocas posibilidades para "encajar", tanto con los oyentes como con los Sordos. Éste ha sido un tema explorado en otras investigaciones (Acosta 2006, de la Paz y Salamanca 2009; Ladd, 2011), en particular, por las diferencias que imprime en la identidad de los sujetos, la manera como enfrentan las barreras para la comunicación, las estrategias que desarrollan para incluirse y sobre todo la defensa que hacen de la Cultura Sorda.

Pero, los entrevistados no son ingenuos, saben que, en un mundo sustentado en la idea de normalidad, sus oportunidades dependerán del manejo que tengan del español. Por esta razón, todos ellos y sus familias pusieron mucho empeño en que fueran a la escuela y aprendieran el español oral y escrito, con la expectativa de que tuvieran mayores posibilidades de cursar una carrera, conseguir un empleo y mejorar su nivel de vida.

Sí, me mandaron a la escuela del auditivo y del lenguaje, mis papás querían tenerme, verme bien. En ese estuve de preescolar hasta la primaria de segundo año. Ahí, me enseñaron a cómo hablar, cómo comprender y el lenguaje de señas, pero yo no usaba la seña mucho, me costaba trabajo. (Entrevistado 3) 
A pesar de esto, la relación que mantienen con el idioma español es ambivalente. Si bien reconocen que éste ha sido un recurso valioso para continuar estudiando, ampliar su red de apoyo y entrar al mercado laboral, de cierta manera, todos se han sentido discriminados por los oyentes o han sido testigos del rechazo que la Comunidad de Sordos tiene hacia aquellos que fueron oralizados y no utilizan la lengua de señas.

Pero, creo que uno de los problemas, es que nos hace falta educación, nos hace falta trabajo, el tema de la discriminación para los sordos, creo que de repente todos los puestos directivos, todos los puestos altos los tienen los oyentes. Digo, yo tuve la fortuna de que mis papás o de una u otra manera, tuvimos dinero y pude poner mi negocio, pero los puestos altos los tienen los oyentes [...] (Entrevistado 2)

Sobre el conflicto al interior de la Comunidad de Sordos, uno de los entrevistados explicó:

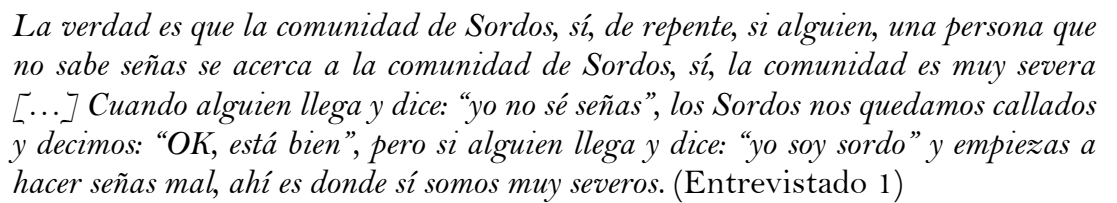

En todos los casos, ingresar al sistema educativo fue una oportunidad altamente valorada, pero, al mismo tiempo, con barreras que requirieron de la voluntad personal y el apoyo de sus familias para superarlas, dado que las instituciones no siempre estaban preparadas para recibirlos. Durante la educación básica, los seis entrevistados hicieron cursos tanto en escuelas regulares como en especiales y fue la que mayores retos les representó; además tres de ellos señalaron que la formación recibida en la primaria y la secundaria había sido muy elemental, lo que les generó dificultades cuando ingresaron a la educación media.

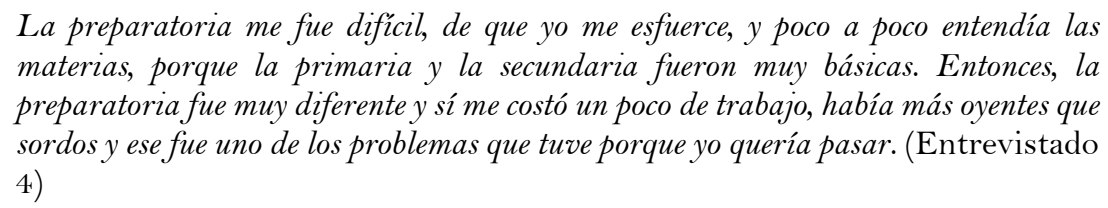

En la educación media, las experiencias fueron más diversas; tres dijeron haber recibido algún tipo de apoyo por parte de sus instituciones, ya sea académico o de accesibilidad a las clases por medio del intérprete.

De repente, yo no podía sola y dije quiero un intérprete y en base a las prácticas pude
sacar la preparatoria. Sí entendía, pero con intérprete, sin él no hubiera podido sacar
la preparatoria [... J No fue un ambiente muy grato, pero, pues, al recibir el apoyo de
los intérpretes y haber aprendido, pues me gustó, pero, fue un poco difícil, bueno, hubo
un poco falta de estudios, ya que no aprendí muy bien. (Entrevistado 5)

Los otros tres no recibieron ningún apoyo, por lo que su trayectoria académica fue más irregular; uno de ellos tuvo que abandonar la preparatoria para retomarla años después y los otros dos enfrentaron dificultades para comprender los contenidos de los cursos, cumplir con las actividades y tareas, y aprobar las materias.

Terminando la secundaria ya no había prepa para sordos. En aquel entonces no había ninguna opción, entonces, volvi a estar en una escuela de oyentes y evidentemente mis compañeros tenían un nivel mucho más alto que el mío y yo no pude concluirlo en ese momento, porque estaba muy atrasado y no pude emparejarme, como persona sorda vas súper atrasado. $\Upsilon$ no pude terminar $y$, pues, se me fue el tiempo, mis compañeros estuvieron ahí, perdí un año de la prepa, después, me volví a meter a la prepa y ya tenía como esta noción de que tenía que irme poniendo al corriente [...] (Entrevistado 1) 
Si la educación obligatoria es una carrera de obstáculos para las personas Sordas, la superior se complica aún más. La primera barrera es encontrar una institución que los acepte y disponga de estrategias de inclusión, ya que, en México, los Sordos tienen una participación muy baja en la educación terciaria, alrededor del $4 \%$ de los 1.4 millones registrados hasta 2018 (INEGI, 2018). Sus oportunidades disminuyen cuando no poseen una alta competencia en español, porque las instituciones no cuentan con intérpretes en lengua de señas.

De las cinco personas que llegaron a este nivel educativo, ninguna tomó la carrera que inicialmente quería, sino que el criterio que siguieron fue inscribirse en la institución que les diera acceso y en el programa que tuvieran más posibilidades de concluir. Pero ésta no es la única barrera, otras señaladas por los entrevistados fueron: la débil formación obtenida en los niveles educativos previos, la carencia de becas de estudio y de apoyos académicos, la poca empatía y capacitación por parte de algunos profesores para trabajar con personas Sordas, la escasez de materiales adecuados y la falta de información sobre la oferta académica accesible.

\section{Me motivó el ingreso aquí porque yo he querido terminar la universidad. Me decían que no había universidades y que a los sordos no nos aceptaban y, cuando supe de aquí, quise seguir adelante y aproveché la oportunidad que me brindaron [...] (Entrevistado 6)}

Sobre los apoyos académicos, un estudiante señaló:

Pues, yo necesito asesoría para poder realizar mi estudio adelante, que me pueda ayudar [en] los temas que se me van dificultando, un apoyo para resolver los problemas, como un guía. (Entrevistado 3)

Un problema reiterado por los entrevistados fue la escasez de intérpretes, ya que por lo general las instituciones no los consideran como parte de los apoyos y tampoco tienen recursos para pagarlos. Como consecuencia, los estudiantes y sus familias tienen que solventarlos, cuando ésta es una obligación de las instituciones públicas establecida en la Ley Federal para Prevenir y Eliminar la Discriminación, vigente en nuestro país (Diario Oficial de la Federación, 2014). Pero, además, en el nivel superior, el intérprete no sólo debe estar certificado en la lengua de señas, sino que tiene que especializarse por campos disciplinarios, lo que eleva considerablemente los costos y disminuye las posibilidades de que las universidades inviertan en contratarlos.

O sea, forzosamente necesito el intérprete, forzosamente, pero ¿̇qué va a pasar si este intérprete no tiene conocimientos de política o de abogacía? To le puedo decir: "aunque tienes certificado" y si me dice que sí, yo voy a pensar: "Ah OK, tiene el certificado, tiene un buen nivel, tiene buenos conocimientos, pero yo no voy a saber si él está cumpliendo con el perfil que yo necesito. (Entrevistado 2)

No obstante, a la par de los obstáculos, cinco de los entrevistados reconocieron el papel de ciertos facilitadores para lograr su permanencia, como las estrategias didácticas utilizadas por algunos los profesores, la disponibilidad del intérprete en lengua de señas, el apoyo de sus compañeros de clases, la buena actitud de los docentes y el respaldo de sus padres.

La atención académica de aquí es buena, el director nos ha dado muchas herramientas, de repente hay algún problema. Aquí, se ha dicho respeten a los sordos en educación, atiéndanlos, nunca nos han tratado mal, nos respetan, para mi es agradable. (Entrevistado 4) 
Con respecto a la actitud de sus pares oyentes, todos señalaron que, aunque al inicio enfrentaron ciertas dificultades de comunicación, ellos siempre tuvieron la disposición para incluirlos como parte del grupo.

Fue difícil comunicarnos porque los alumnos no sabían lengua de señas y que posteriormente les dice a los oyentes que yo era sorda y no me hicieron nada, fueron muy buenos conmigo, me ayudaron y me explicaban como podían y también me decía que querían tomar clases de señas para poder apoyarme y eso me agradó mucho. (Entrevistado 5)

Vale la pena destacar que, aunque la mitad de los estudiantes reconoció la buena actitud de los profesores para apoyarlos en sus clases, sólo dos aludieron de manera específica a las estrategias didácticas, como el uso del pizarrón y la evaluación accesible.

\begin{abstract}
Las clases que dan los maestros explican muy bien, me gusta cómo a nosotros siempre nos anotan las cosas en el pizarrón para que podamos copiar y lo que están explicando. Hay algo difícil como todo, pero nos apoyan para que podamos seguir la clase. (Entrevistado 6)
\end{abstract}

Finalmente, queremos señalar que, para todos nuestros sujetos de estudio, la educación constituye un recurso muy valioso en contra de la discriminación. Éste es un tema en el que coincidieron los seis entrevistados y que, de alguna manera, refleja las barreras que han tenido que enfrentar en una sociedad que, históricamente, ha tratado de negar la diversidad humana (Parrilla, 2002), con prácticas que van desde la normalización hasta la exclusión simbólica o real de los considerados diferentes.

\begin{abstract}
[... la verdad es que, como te decir, bien claro, a mí me parece que en, este, en la comunidad de sordos, es como si estuviéramos en los noventa, no tenemos, no ha llegado la suficiente información y pues sí, parece discriminación, pero, obedece más bien a que no se sabe, no hay información. (Entrevistado 1)
\end{abstract}

\title{
7. Consideraciones finales
}

La exclusión es una problemática generalmente asociada a ciertos grupos en situación de vulnerabilidad, sin embargo, parafraseando a Karsz (2004), podríamos decir que, en los Sordos, ésta tiene itinerarios específicos que pasan por la tensión entre su reconocimiento como personas con discapacidad, como comunidad lingüístico-cultural o, incluso, como un conjunto de comunidades. En este artículo, partimos de dicho debate para analizar las experiencias de inclusión y exclusión social de un grupo de Sordos, usuarios de la LSM.

Fundamentalmente, nos interesaba destacar tres dimensiones. La primera tiene que ver con el proceso de construcción identitaria. En nuestra investigación, encontramos que la manera de asumir la sordera difirió significativamente entre los que crecieron en una familia de Sordos, para quienes no escuchar fue parte de la normalidad hasta que ingresaron a la escuela, y los que crecieron en una familia de oyentes, en donde la sordera era vista como una discapacidad. La importancia otorgada a la lengua de señas o a la oral marca de manera preponderante, aunque no única, la identidad en las personas Sordas. De este modo, algunas tienden a verse a sí mismas como enfermas y la oralidad es su referente de normalidad a alcanzar; otras tienen una especie de "identidad marginal", ya que no se sienten parte ni de los Sordos ni de los oyentes; en otros casos hay un fuerte sentido de pertenencia a la comunidad de Sordos, así como con el uso de la lengua de señas, que contrasta con el rechazo hacia todo lo relacionado con la oralidad; y, finalmente, están aquellos que tienen una identidad bicultural, porque aunque se sienten Sordos, valoran positivamente el universo de los oyentes (Goldblat y Most, 2018). 
En cualquiera de los casos, la lengua y la comunicación representa un verdadero reto para ellos, no sólo porque la mayor parte del tiempo están inmersos en contextos de oyentes y hablantes, sino porque, además, son personas a quienes tácita o explícitamente se les atribuye una condición de excepcionalidad, debido a que no son competentes en la lengua dominante, lo que repercute en el ejercicio de sus derechos y en su participación social.

La segunda dimensión gira en torno a la identidad colectiva. Para la mayoría de nuestros entrevistados, ser Sordo significó ser parte de una comunidad con formas de interacción, normas, valores y tradiciones que merecen ser reconocidos positivamente y que les permiten situarse en el mundo de manera distinta a como lo hacen los oyentes (de la Paz y Salamanca, 2009). Sin embargo, los Sordos no son una población homogénea; en su interior existen distintas culturas en las que se entrecruzan otros referentes identitarios, como el género, la edad, la etnia, etcétera. Estas culturas no siempre marchan al mismo ritmo, ni comparten los mismos intereses, como en nuestros sujetos de estudio, quienes reconocieron la diversidad y los conflictos existentes al interior de su comunidad, así como el rechazo hacia aquellos que, siendo Sordos, no tienen una buena competencia en la LSM. "Las interseccionalidades de estas identidades abarcan grados variables de influencias estigmantizantes u optimizadoras, que pueden determinar el valor atribuido a cada constelación de identidades" (Leigh, 2019, p. 6).

La siguiente dimensión nos remite a la compleja relación que estas personas mantienen con el español y la lengua de señas. En nuestra investigación, todos los entrevistados eran señantes $\mathrm{y}$, con excepción de uno, todos consideraban a esta última como su primera lengua y como un rasgo fundamental de su identidad. Igualmente, aunque concedieron que haber aprendido español les había representado ciertas ventajas, para la mayoría la oralización fue un proceso muy difícil sobre el que no siempre tuvieron opción, lo que refleja el peso que culturalmente se le ha dado a la voz como medio de comunicación y como indicador de la inteligencia (Rose, 2006). Además, contribuye a reforzar la idea de que los Sordos tienen que ser "curados" o "rehabilitados" a través del implante coclear, terapias auditivo-verbales o la educación especial.

En general, la situación para los Sordos es compleja porque las estrategias oralizadoras parecen olvidar que la relación entre audición y habla no es causal, al contrario, en el lenguaje hablado intervienen múltiples factores, que van desde la personalidad de los individuos hasta el entorno en donde se lleva a cabo la comunicación, incluso, están en juego unidades de significado que entrañan información adicional a la que se intercambia en un determinado momento (Leigh, 2012). De modo que, a diferencia de lo que comúnmente se piensa, lograr una adecuada competencia lingüística no sólo depende de la cantidad de inputs orales o del nivel de audición de los sujetos, sino que se trata de un proceso mucho más elaborado.

La cuarta dimensión tiene que ver con la trayectoria educativa. Aquí, las experiencias de las personas Sordas se asemejan a las de otros individuos en situación de vulnerabilidad, que enfrentan múltiples barreras para ingresar, mantenerse y egresar de cada uno de los niveles de enseñanza. El predominio de la educación especial como opción formativa para los Sordos ha sido un factor muy importante. En nuestros sujetos de estudio, sus familias tomaron la decisión de enviarlos a este tipo de escuelas para oralizarlos. Sin negar el aporte que estos profesionales pueden hacer al desarrollo de los estudiantes, la educación especial no puede continuar siendo la única modalidad para los Sordos, ni para nadie, sino que los 
sistemas educativos deben crear las condiciones para que sean incluidos en la enseñanza regular.

Particularmente, en México, el modelo bilingüe-bicultural ha fracasado en su cometido inicial de favorecer a la inclusión de los Sordos, ya que, por un lado, invierte gran parte de sus esfuerzos en el aprendizaje del español oral y escrito, mientras que, por otro, no logra que los alumnos adquieran los conocimientos y habilidades necesarias para su desarrollo personal. Se trata de un claro ejemplo de inclusión excluyente, en donde el sistema educativo abre formalmente los espacios para que estas personas tengan acceso a las instituciones, pero, parece tener poco interés en generar condiciones para que permanezcan, aprendan y continúen avanzado hacia los siguientes niveles educativos. Además, para la Comunidad de Sordos, tendría que reconocerse que la lengua de señas es su primera lengua y ésta es la que los estudiantes deberían utilizar y perfeccionar durante sus primeros años de formación, para, posteriormente, enseñarles la lengua dominante de su cultura (Becerra, 2008; Lissi et al., 2012), como se ha hecho, por ejemplo, en las personas de los pueblos originarios. Sin embargo, en esta población, se da por sentado que, si quieren ingresar a la educación regular, tienen que ser oralizados.

Por otra parte, si bien el enfoque de la inclusión ha impulsado el desarrollo de nuevos modelos educativos, todavía se hacen importantes esfuerzos por "normalizar" a los estudiantes Sordos, lo cual incide tanto en el acotado manejo que muchos de ellos tienen tanto de la lengua oral y escrita, como en su formación académica, que suele ser más baja que la de sus pares oyentes (Hyde et al., 2016). Éste fue un problema señalado por nuestros entrevistados, quienes junto con sus familias entendieron desde muy temprano que, si querían ampliar sus oportunidades educativas, necesitaban aprender español. A pesar de ello, todos enfrentaron muchas dificultades al ingresar a la enseñanza regular debido a que su formación en la educación especial había sido muy limitada, de manera que, además de lidiar con las barreras de la comunicación, tuvieron que invertir más tiempo y esfuerzo para responder a las exigencias académicas.

El predominio del oralismo se manifiesta a lo largo de todo el sistema educativo, minando el reconocimiento de la LSM como primera lengua de este colectivo, aunque en nuestro país la ley proscribe cualquier discriminación, exclusión, restricción o preferencia por motivos de lengua o idioma. Lo anterior también ha contribuido a la creación de una serie de cotos por los que el saber oyente se ha impuesto a una minoría con historia, valores y cultura propios, los cuales han sido documentados en la academia, pero, muchas veces son ignorados por las instituciones públicas o subsumidos en políticas y programas diseñados para otros colectivos sociales.

Por si esto fuera poco, conforme se va avanzando en los niveles educativos, las opciones educativas para los Sordos se reducen aún más. Por ejemplo, en las personas entrevistadas, como todos cursaron los estudios básicos en el sistema de educación especial no enfrentaron problemas importantes para la comunicación con sus maestros y compañeros, pero, al llegar a la preparatoria, sólo la mitad pudo disponer del servicio del intérprete en lengua de señas, mientras que la otra mitad se las tuvo que arreglar para estudiar y concluirla sin este apoyo. Esto les representó múltiples obstáculos para completar las actividades y trabajos en clase, aprobar las asignaturas y llevar una trayectoria escolar continua. Aquí, vale la pena señalar que, sin dudas, la disponibilidad de un intérprete en lengua de señas es un recurso importante para la inclusión de los alumnos Sordos, pero, no es el único, las instituciones educativas necesitan diseñar otras estrategias que 
favorezcan su éxito académico, como la formación de los docentes para trabajar con grupos cada vez más diversos, la implementación de ajustes para la enseñanza, la distribución de apoyos académicos y económicos, la sensibilización de los miembros de la comunidad institucional y la capacitación de los intérpretes en los contenidos disciplinarios.

En el nivel superior, la situación no es muy diferente. La poca información sobre la oferta y los servicios institucionales, la escasez de apoyos económicos y académicos, la falta de profesores capacitados y la carencia de materiales adaptados fueron las barreras más importantes para nuestros entrevistados. "Estos factores pueden influir significativamente en su participación académica y social, en el rendimiento de los cursos en el nivel superior y, en última instancia, en la conclusión del programa” (Hyde et al., 2016, p. 442). Todas estas problemáticas no son exclusivas de un determinado sistema educativo, al contrario, la Organización Mundial de la Salud (OMS, 2011) ha denunciado que las personas con dificultades para la comunicación tienden a enfrentar múltiples desventajas sociales, tanto en los países en desarrollo como en los desarrollados, especialmente en los ámbitos de la atención médica, el gobierno local, la justicia y, por supuesto, la educación.

No obstante, a la par de las barreras, están los facilitadores en los que también contribuyen los diferentes actores educativos, profesores, estudiantes, directivos y padres de familia. En nuestros entrevistados, por ejemplo, estos últimos se convirtieron en el motor más importante para terminar la educación superior, porque no sólo los impulsaron a seguir adelante, sino que los ayudaron económica y académicamente, cuando les fue posible. También, recibieron el apoyo de sus compañeros para incluirlos en las clases y de los docentes quienes, en algunos casos, ajustaron sus estrategias didácticas y de evaluación para hacerlas accesibles.

Finalmente, queremos insistir en el compromiso que deben asumir las instituciones para crear condiciones que permitan a los Sordos ejercer su derecho a la educación. La inclusión no tiene que depender de la buena voluntad de los profesores o de las autoridades educativas, sino que se deben diseñar políticas de largo plazo que contrarresten los factores que incrementan el riesgo de exclusión, reconozcan la diversidad del alumnado y en las que más que tratar de importar modelos pensados para otros contextos, se sustenten en el conocimiento experto que los Sordos tienen de sus necesidades y de la realidad que viven cotidianamente.

\section{Referencias}

Acosta, V. (2006). Perspectivas en el discurso de la sordera. En V. Acosta (Dir.), La sordera desde la diversidad cultural y lingüística (pp. 1-26). Masson.

Antia, S., Knoors, H. y Marschark, M. (2019). Co-enrollment and the education of deaf and hardof-hearing learners: Foundations, implementation, and challenges. En M. Marschark, A. Shirin y H. Knoors (Eds.), Co-enrollment in deafeducation (pp. 1-24). Oxford University Press.

Barreto, A. y Cortés, Y. (2014). Aspectos relevantes del discurso en Lengua de Señas Colombiana. En S. Soler, D. Calderón y A. Ferroni (Eds.), Panorama de los estudios del discurso en Colombia (pp. 245-281). Universidad Distrital Francisco José de Caldas.

Baynton, D. (2006). A silent exile on this earth. The metaphorical construction of deafness in the Nineteenth century. En L. Davis (Ed.), The disability studies reader (pp. 33-48). Routledge.

Becerra, C. (2008). Lenguaje y educación en niños sordos: encuentros y desencuentros. REXE. Revista de Estudios y Experiencias en Educación, 14, 105-114. 
Blanco, R. (2008). Haciendo efectivo el derecho a una educación de calidad sin exclusiones. Revista Colombiana de Educación, 54, 14-35.

Castel, R. (2004). Encuadre de la exclusión. En S. Karsz (Coord.), La exclusión: Bordeando sus fronteras. Definiciones y matices (pp. 55-86). Gedisa.

Cuevas, H. (2013). El gobierno de los sordos: El dispositivo educacional. Revista de Ciencia Política, 33(3), 693-713. https://doi.org/10.4067/So7 18-090X2013000300006

Davis, L. (1995). Enforcing normalcy. Disability, deafness and the body. Verso.

Davis, L. (2006). Constructing normalcy: The bell curve, the novel, and the invention of the disabled body in the nineteenth century. En L. Davis (Ed.), The disability studies reader (pp. 3-16). Routledge.

De la Paz, M. y Salamanca, M. (2009). Elementos de la cultura sorda: Una base para el currículum intercultural. REXE. Revista de Estudios y Experiencias en Educación, 8(15), 31-49.

Del Castillo, A. (2005). Médicos y pedagogos frente a la degeneración racial: la niñez en la ciudad de México, 1876-1911. En C. Agostoni y E. Speckman (Eds.), De normas y transgresiones: Enfermedad y crimen en América Latina (1850-1950) (pp. 83-107). IIH / UNAM.

Diario Oficial de la Federación. (2014). Decreto por el que se reforman, adicionan y derogan diversas disposiciones de la Ley Federal para prevenir y eliminar la discriminación. Diario Oficial de la Federación.

Goldblat, E. y Most, T. (2018). Cultural identity of young deaf adults with cochlear implants in comparison to deaf without cochlear implants and hard-of-hearing young adults. Journal of Deaf Studies and Deaf Education, 23(3), 228-239.

Herrera, V., Chacón, D. y Saavedra, F. (2016). Evaluación de la escritura de estudiantes sordos bilingües. Estudios Pedagógicos, 42(2), 171-191. https://doi.org/10.4067/S07 18-07052016000200010

Hyde, M., Nikolaraizi, M., Powell, D. y Stinson, M. (2016). Critical factors toward the inclusion of Deaf and Hard-of-Hearing students in higher education. En M. Marschark, V. Lampropoulou y E. Skordilis (Eds.), Diversity in deaf education (pp. 441-472). Oxford University Press.

Instituto Nacional de Estadística y Geografía. (2018). Encuesta nacional de la dinámica demográfica 2018. Microdatos. INEGI.

Karsz, S. (2004). La exclusión: Concepto falso, problema verdadero. En S. Karsz (Coord.), La exclusión: Bordeando sus fronteras. Definiciones y matices (pp. 133-214). Gedisa.

Ladd, P. (2011). Comprendiendo la cultura sorda. En busca de la sordera. Consejo Nacional de la Cultura $\mathrm{y}$ las Artes.

Leigh, I. (2012). Not just deaf: Multiple intersections. En R. Nettles y R. Balter (Eds.), Multiple minority identities (pp. 59-80). Springer.

Leigh, I. (2019). Deaf identities: A maturing framework. En I. Leigh y C. O’Brien (Eds.), Deaf identities. Exploring new frontiers (pp. 1-26). Oxford University Press.

Liamputtong, P. (2010). Performing qualitative cross-cultural research. Cambridge University Press.

Lissi, M., Svartholm, K. y González, M. (2012). El enfoque bilingüe en la educación de sordos. Sus implicancias para la enseñanza y aprendizaje de la lengua escrita. Estudios Pedagógicos, 38(2), 299-320. https://doi.org/10.4067/S0718-07052012000 200019

Martín-Crespo, M. y Salamanca, A. (2007). El muestreo en la investigación cualitativa. Nure Investigación, 27, 1-4. 
Martínez, M. (2012). Actores de la educación especial en México: Representaciones y prácticas sociales en la segunda mitad del siglo XX. En A. Padilla (Coord.), Arquetipos, memorias y narrativas en el espejo: Infancia anormal y educación especial en los siglos XIX y XX (pp. 269-306). UAEM.

Moores, D. (2010). The history of language and communication issues in deaf education. En M. Marschark y P. E. Spencer (Eds.), The Oxford handbook of deaf studies, language and education (pp. 17-30). Oxford University Press.

Organización Mundial de la Salud. (2011). Informe mundial sobre la discapacidad. OMS.

Parrilla, A. (2002). Acerca del origen y sentido de la educación inclusiva. Revista de Educación, 237, 11-29.

Padilla, A. (2010). La educación especial en México a finales del siglo XIX y principios del XX: Ideas, bosquejos y experiencias. Revista Educación y Pedagogía, 22(57), 15-29.

Rose, M. L. (2006). Deaf and dumb in ancient Greece. En L. Davis (Ed.), The disability studies reader (pp. 17-31). Routledge.

Saizarbitoria, R. (1999). La elección del sistema de comunicación en la educación de los niños sordos. Zerbitzuan, 23, 58-66.

Skliar, C. (1998). Bilingüismo e biculturalismo. Uma análise sobre as narrativas tradicionais na educação dos surdos. Revista Brasileira de Educação, 8, 44-57.

Skliar, C. (2012). Os estudos surdos em educação: Problematizando a normalidade. En C. Skliar (Org.), A surdez. Um olhar sobre as diferenças (pp. 7-32). Mediaçao.

Subirats, J., Gomá, R. y Brugué, J. (2005). Análisis de los factores de exclusión social. Fundación BBVA.

Thompson, P. (1988). La voz del pasado. La historia oral. Edicions Alfons El Magnanim.

\section{Breve CV de los/as autores/as}

\section{Judith Pérez-Castro}

Doctora en Ciencia Social con especialidad en Sociología por El Colegio de México. Investigadora de tiempo completo en el Instituto de Investigaciones sobre la Universidad y la Educación (IISUE) de la Universidad Nacional Autónoma de México (UNAM). Miembro del Sistema Nacional de Investigadores (SNI) nivel 2, del Consejo Nacional de Ciencia y Tecnología (CONACYT). Sus líneas de investigación son: Equidad e inclusión educativa, Políticas educativas, Ética profesional y valores profesionales. Entre sus publicaciones recientes están: "Acceso a la educación y factores de vulnerabilidad en las personas con discapacidad" y "Condiciones para la docencia inclusiva: análisis de las barreras y los facilitadores". ORCID ID: https://orcid.org/0000-0002-5176-0531. Email: pkjudith33@yahoo.com.mx

\section{Johan Cristian Cruz-Cruz}

Programa de Becas Posdoctorales de la Universidad Nacional Autónoma de México (UNAM), en el Instituto de Investigaciones sobre la Universidad y la Educación (IISUE). Doctor en Educación por la Universidad Autónoma del Estado de Morelos, México. Candidato a Investigador Nacional del Sistema Nacional de Investigadores, del Consejo Nacional de Ciencia y Tecnología. Sus líneas de investigación son: Historia del cuerpo y corporeidades, Procesos culturales en la Comunidad de Sordos, Representaciones sociales 
y discapacidad. Sus publicaciones recientes están "El sordo como sujeto emergente ante procesos de vulnerabilidad y equidad en el nivel superior: miradas, practicas documentadas y representaciones", "Corporeidad, lengua y educación. El caso de las Comunidades Sordas". ORCID ID: https://orcid.org/0000-0002-8844-0574. Email: johanccruz@gmail.com 